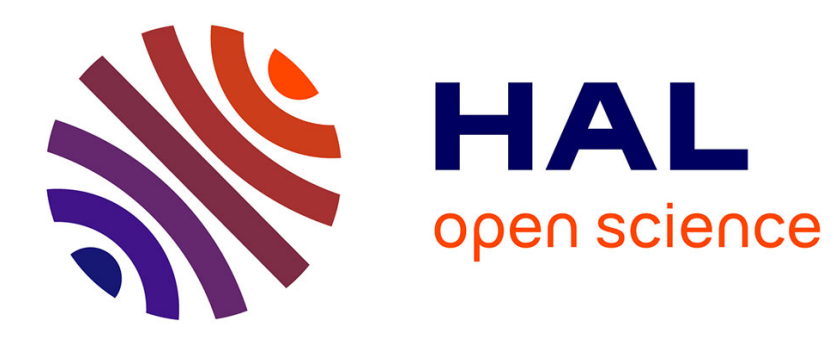

\title{
Modélisation des mécanismes de conduction non linéaires dans les varistances $\mathrm{ZnO}$ \\ P. Manuel
}

\section{To cite this version:}

P. Manuel. Modélisation des mécanismes de conduction non linéaires dans les varistances ZnO. Revue de Physique Appliquée, 1987, 22 (9), pp.971-983. 10.1051/rphysap:01987002209097100 . jpa00245657

\section{HAL Id: jpa-00245657 https://hal.science/jpa-00245657}

Submitted on 1 Jan 1987

HAL is a multi-disciplinary open access archive for the deposit and dissemination of scientific research documents, whether they are published or not. The documents may come from teaching and research institutions in France or abroad, or from public or private research centers.
L'archive ouverte pluridisciplinaire HAL, est destinée au dépôt et à la diffusion de documents scientifiques de niveau recherche, publiés ou non, émanant des établissements d'enseignement et de recherche français ou étrangers, des laboratoires publics ou privés. 


\title{
Modélisation des mécanismes de conduction non linéaires dans les varistances $\mathrm{ZnO}$
}

\author{
P. Manuel \\ Direction des Etudes et Recherches, Electricité de France, \\ 1, avenue du Général de Gaulle, 92141 Clamart, France
}

(Reçu le 8 décembre 1986, accepté le 18 mai 1987)

\begin{abstract}
Résumé. - Une méthode de calcul de la caractéristique courant-tension des varistances à l'oxyde de zinc a été développée. Elle fait appel aux modèles courants de double barrière de Schottky et d'émission thermoionique dans la zone des faibles courants où le coefficient de non-linarité $\alpha$ est inférieur à 30. La partie originale du modèle réside dans le traitement de la zone des courants intermédiaires, qui correspond aux plus fortes valeurs de $\alpha$. On invoque un mécanisme d'ionisation d'impact initié par des électrons de forte énergie pour expliquer l'apparition d'un courant de trous dirigé vers l'interface entre deux grains d'oxyde de zinc. La probabilité d'ionisation est calculée par une méthode de Monte Carlo et comparée à des relations analytiques qui constituent un minorant et un majorant du résultat. La recombinaison des trous est traitée moyennant un certain nombre d'hypothèses sur la position en énergie et la section efficace de capture des états localisés à l'interface. La charge positive résultant du piégeage des trous à l'interface provoque un abaissement brutal des barrières de potentiel entre grains d'oxyde de zinc. Le modèle prévoit des coefficients de non-linéarité supérieurs à 60 , en accord avec l'observation.
\end{abstract}

\begin{abstract}
A calculation method has been developed for the current-voltage characteristic of zinc-oxide varistors. This method calls for the standard models of double Shottky barrier and thermionic emission in the low current range where the non-linearity coefficient $\alpha$ is below 30 . The novel part of the model lies in the treatment of the intermediate current range which corresponds to the highest values of $\alpha$. An impact ionization mechanism generated by high-energy electrons is put forward to explain the appearance of a hole current directed to the interface between two zinc-oxide grains. The ionization probability is calculated by a MonteCarlo method and compared with analytic relations which form both a lower bound and an upper bound for the result. Hole recombination is taken into account using a number of assumptions on the energy distribution and the capture cross-section of the localized states at the interface. The positive charge resulting from hole trapping at the interface brings about a sudden lowering of the potential barriers between zinc-oxide grains. The model provides nonlinearity coefficients higher than 60 , in agreement with the experiments.
\end{abstract}

\section{Introduction.}

De nombreux modèles ont été développés pour rendre compte des propriétés électriques non linéaires des céramiques à base d'oxyde de zinc. Dès les premières études [1-4], l'origine de la non-linéarité a été attribuée aux zones situées à la périphérie des grains de $\mathrm{ZnO}$. Aucun champ électrique notable n'apparaît au cœur de ceux-ci, sauf aux très forts courants.

Les études de microstructure ont révélé que les phases secondaires présentes en faibles quantités laissent dans la plupart des cas les grains de $\mathrm{ZnO}$ en contact direct avec leurs voisins. L'épaisseur d'une éventuelle couche intergranulaire, indétectable en microscopie électronique, est alors au plus de l'ordre de quelques $\mathrm{nm}$. Ces zones de contact seront appelées interfaces dans la suite de ce travail.

La plupart des modèles récents partent d'une description commune de la structure de bandes au voisinage des interfaces. Celles-ci sont porteuses d'états localisés profonds dont le bilan de charge est négatif. Il en résulte l'apparition d'une barrière de potentiel que l'on peut assimiler à un assemblage de deux jonctions Schottky placées dos à dos [5-10] (Fig. 1a).

Des différences notables subsistent encore quant au traitement de cette double barrière de Schottky (DBS) lorsqu'elle est soumise à une différence de potentiel. Selon certains travaux $[3,5,8]$, l'augmen- 


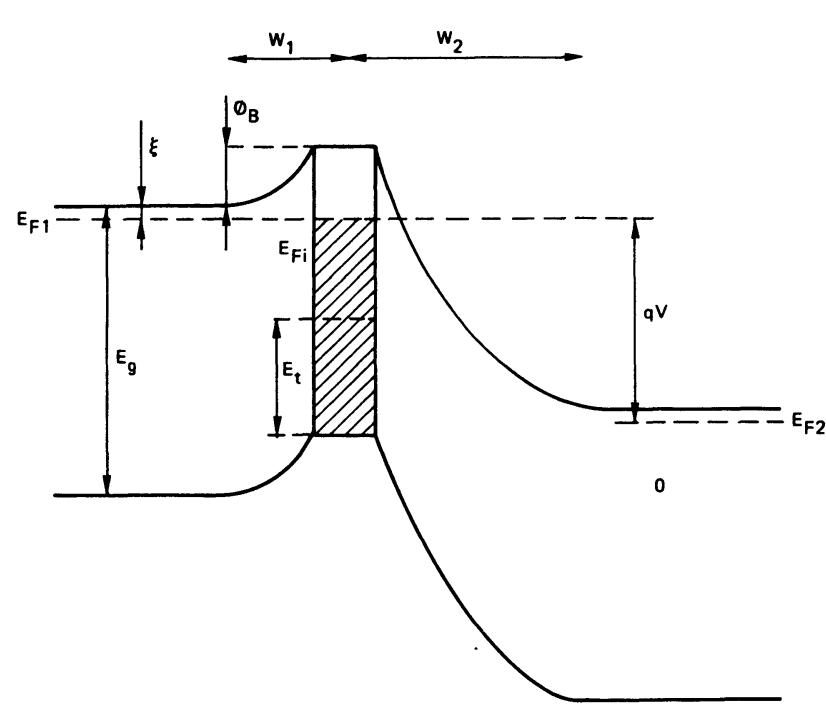

Fig. 1a. - Schéma de bandes au voisinage d'un interface entre deux grains d'oxyde de zinc. Modèle de la double barrière de Schottky pour une polarisation $V>V_{s}$. En hachuré : états occupés de l'interface. (L'épaisseur de l'interface proprement dit est supposée négligeable.)

[Energy-band diagram around an interface between two zinc-oxide grains. Double Shottky barrier model for a polarization $V<V_{s}$. Hatched : occupied states of the interface (the real interface thickness is assumed to be negligible).]

tation ra ide

dessus d'un seuil de tension $V_{\mathrm{s}}$ est essentiellement liée à la forme de la densité d'états localisés présents à l'interface. Ces modèles rencontrent des difficultés pour expliquer l'indépendance relative de $V_{\mathrm{s}}$ vis-àvis du processus de fabrication $[11,12]$.

A la suite de ces observations, une nouvelle classe de modèles reliant la valeur de $V_{\mathrm{s}}$ à la largeur de bande interdite $E_{\mathrm{g}}$ de l'oxyde de zinc a été élaborée $[7,9]$. Ils attribuent la variation de $\alpha$ au voisinage de $V_{\mathrm{s}}$ à l'apparition d'un processus de création de paires électron-trous dans la zone désertée polarisée en inverse de la DBS. (Ceci nécessite approximativement $q V_{\mathrm{s}}>E_{\mathrm{g}}$.) Sous l'effet du champ électrique local, les trous sont collectés au voisinage de l'interface, dont le bilan de charge se trouve alors brutalement perturbé. L'affaiblissement des barrières de potentiel qui en résulte permet un accroissement très rapide du courant pour une faible variation de tension.

Dans cet ordre d'idées deux modèles ont été proposés. Le premier suppose que les trous restent confinés du côté du grain polarisé positivement, et que leur distribution en énergie soit déterminée par la position du niveau de Fermi loin de l'interface [7]. L'intérêt de ce modèle est de comporter très peu de paramètres ajustables et de fournir une prédiction correcte de la valeur de $\alpha$. Aucune justification n'est cependant fournie en ce qui concerne le confinement asymétrique des trous. Ce modèle néglige par ailleurs l'influence des recombinaisons avec les électrons, alors que celles-ci jouent habituellement un rôle essentiel dans l'établissement d'un équilibre dynamique de cette nature.

Un second modèle prend partiellement en considération ce processus de recombinaison, mais il ne traite que de façon qualitative le mécanisme de création des paires électron-trous [9]. Il conduit à un coefficient de non-linéarité égal à 30 , ce qui est inférieur aux valeurs observées.

On a tenté dans cette étude de compléter les deux approches précédentes en fournissant une évaluation plus quantitative des coefficients de non-linéarité que l'on peut obtenir par ce type de modèle. On a effectué dans ce but un traitement aussi complet que possible du mécanisme de création de paires électron-trou. En ce qui concerne l'influence des recombinaisons à l'interface, plusieurs hypothèses sur la nature du processus dominant ont été considérées. Cependant, seule celle qui nous a fourni le meilleur accord pour les caractéristiques courant-tension des varistances sera présentée dans cet article. Elle diffère notablement du traitement effectué par Pike [9].

Il n'est bien évidemment pas question de tenter d'obtenir un accord très précis du modèle avec des résultats expérimentaux obtenus sur des varistances massives : les irrégularités du matériau, la variabilité nnaissance de nombreux paramètres physiques constituent des obstacles probablement insurmontables à cet égard. Il s'agit plutôt de montrer que l'attribution de valeurs vraisemblables à ces paramètres est compatible avec la prédiction de coefficients $\alpha$ égaux ou supérieurs à ceux qui sont observés.

Des résultats partiels concernant ce modèle ont déjà été publiés [13]. Par ailleurs, une autre équipe a également publié des résultats préliminaires obtenus par un modèle voisin [14].

\section{Présentation du modèle.}

2.1 HYPOTHÈSES CONCERNANT LES PROPRIÉTÉS DES ÉTATS LOCALISÉS A L'INTERFACE. - Contrairement au traitement effectué par Pike [9], on considère que les trous drainés vers l'interface sont immédiatement piégés par les centres profonds qui lui sont associés. Ce point de vue est justifié par le fait que les fortes densités d'états localisés que l'on suppose présentes à l'interface laissent présager un temps de piégeage inférieur à $10^{-8} \mathrm{~s}$. Compte tenu des choix de paramètres effectués au paragraphe 2 , ce délai est toujours négligeable par rapport au temps de recombinaison des trous piégés à l'interface avec les électrons de conduction.

La validité du modèle utilisé nécessite que soient vérifiées les trois conditions suivantes : 
- Le courant de réémission des trous collectés à l'interface vers le grain polarisé négativement doit être faible par rapport au courant de recombinaison avec les électrons de la bande de conduction. Ceci est le cas dans la mesure où l'on peut vérifier :

$$
\frac{v_{\text {th }}^{\prime} \sigma_{\mathrm{t}}^{\prime} N_{\mathrm{v}}}{v_{\mathrm{th}} \sigma_{\mathrm{t}} N_{\mathrm{c}}} \exp -\frac{E_{\mathrm{t}}-\xi}{k T} \ll 1 .
$$

Dans cette expression, $N_{\mathrm{v}}$ et $N_{\mathrm{c}}$ représentent les densités d'états effectives des bandes de valence et de conduction, $v_{\text {th }}$ et $v_{\text {th }}^{\prime}$ les vitesses thermiques des électrons et des trous, $E_{\mathrm{t}}$ est l'énergie du piège mesurée au-dessus du sommet de la bande de valence à l'interface. Les sections efficaces $\sigma_{\mathfrak{t}}^{\prime}$ et $\sigma_{t}$, supposées indépendantes de $E_{t}$, concernent respectivement la capture d'un trou par un piège de l'interface, et la capture d'un électron de la bande de conduction (par un tel piège ayant préalablement capturé un trou). $\xi$ est la différence d'énergie entre le niveau de Fermi et la bande de conduction dans les zones neutres. On montre aux paragraphes suivants que $\xi$ est voisin de $0,13 \mathrm{eV}$. Il en résulte que la condition précédente est vérifiée pour la quasitotalité des énergies de piège possibles (la largeur de bande interdite $E_{\mathrm{g}}$ de l'oxyde de zinc est de $3 \mathrm{eV}$ ). L'hypothèse proposée apparaît donc raisonnable.

- On fait l'hypothèse que chaque piège ne possède qu'un seul état correspondant à un niveau d'énergie situé dans la bande interdite. Par ailleurs, les processus de transfert de charge entre pièges voisins de l'interface sont supposés négligeables parce que beaucoup plus lents que les processus de recombinaison entre électrons de conductions et trous piégés. On montre au paragraphe 2.4 que l'on obtient ainsi une distribution de trous à l'interface très éloignée de l'équilibre thermique. Dans l'état actuel des connaissances concernant les états d'interface des varistances, il est difficile de discuter cette hypothèse $\mathrm{du}$ point de vue théorique. On peut seulement mentionner le résultat de calculs que nous avons effectués en prenant l'hypothèse contraire (mise en équilibre thermique des trous piégés à l'interface) : les coefficients de non-linéarité obtenus au voisinage de $V_{\mathrm{s}}$ sont plus faibles et les caractéristiques courant-tension modélisées moins proches des caractéristiques expérimentales que dans le cas des résultats présentés ci-dessous.

- Les états localisés de l'interface sont supposés uniformément distribués en énergie dans la bande interdite.

En fait, cette condition n'est nécessaire à la validité du modèle qu'en ce qui concerne les états situés entre le niveau de Fermi de l'interface sous polarisation nulle et la bande de conduction. Soit $N_{\mathrm{i}}(E)$ cette densité d'états. L'hypothèse adoptée $N_{\mathrm{i}}(E)=$ Cte est relativement neutre puisque l'erreur effectuée sur $\alpha$ change de signe selon que $\mathrm{d} N_{\mathrm{i}} / \mathrm{d} E$ est positif ou négatif [15].

\subsection{MODĖLE DE BANDES AU VOISINAGE DE L'INTER-} FACE. - Les paragraphes 2.2 et 2.3 constituent essentuellement un rappel des modèles de DBS couramment utilisés pour décrire le comportement des joints de grains dans les semiconducteurs.

Les zones désertées sont modélisées dans le cadre des hypothèses simplificatrices suivantes :

- la charge d'espace $\rho(x)$ est constituée d'un seul type de donneur ionisé, dont la concentration volumique $N_{\mathrm{d}}$ est constante au voisinage de l'interface ;

- la charge d'espace s'annule brutalement aux frontières des zones désertées de largeurs $W_{1}$ et $W_{2}$ :

$$
\begin{aligned}
& \rho(x)=q N_{\mathrm{d}} \text { pour }-W_{1}<x<W_{2} \\
& \rho(x)=0 \quad \text { pour } \quad x<-W_{1} \text { ou } x>W_{2}
\end{aligned}
$$

$q$ étant la valeur absolue de la charge de l'électron.

Dans ces conditions, le comportement du modèle en présence d'une différence de potentiel $V$ est décrit par la relation :

$$
V=\frac{Q}{\varepsilon}\left[\frac{Q}{2 q N_{\mathrm{d}}}-\frac{2 \varepsilon \phi_{\mathrm{B}}}{q^{2} N_{\mathrm{d}}}\right]
$$

$Q$ étant la charge électrique piégée à l'interface, $\varepsilon$ la constante diélectrique de $\mathrm{ZnO}$ et $\phi_{\mathrm{B}}$ la hauteur de barrière du côté du grain polarisé négativement. Pour calculer $V$ à partir de $\phi_{\mathrm{B}}$ il est nécessaire d'introduire une relation supplémentaire entre $Q$ et $\phi_{\mathrm{B}}$. Dans le présent modèle, on distingue deux cas selon que $V$ est inférieur ou supérieur à la tension de seuil $V_{\mathrm{s}}$ au-dessus de laquelle des trous peuvent être engendrés par ionisation d'impact.

2.3 COMPORTEMENT AU-DESSOUS DE $V_{\mathrm{s}}$ - On peut montrer à partir de considérations simples [3] que la distribution des charges piégées à l'interface est donnée par une loi de Fermi-Dirac correspondant à un niveau de Fermi $E_{\mathrm{Fi}}$ pratiquement égal à celui du grain polarisé négativement $\left(E_{\mathrm{F} 1}\right)$. On peut alors écrire :

$$
Q=Q_{0}+\left(\phi_{\mathrm{B}_{0}}-\phi_{\mathrm{B}}\right) N_{\mathrm{i}}
$$

La charge piégée sous polarisation nulle $Q_{0}$ étant reliée à la hauteur de barrière correspondante $\phi_{\mathrm{B}_{0}}$ par :

$$
Q_{0}=\left(8 \varepsilon \phi_{\mathrm{B}_{0}} N_{\mathrm{d}}\right)^{1 / 2}
$$

Pour calculer la caractéristique densité de couranttension $J(V)$, il reste à déterminer le courant résultant d'un profil de bandes donné. On utilise pour cela une loi d'émission thermoionique simple : 
$J_{\mathrm{e}}=A^{*} T^{2}\left[\exp -\frac{\phi_{\mathrm{B}}+\xi}{k T}-\exp -\frac{\phi_{\mathrm{B}}+\xi+q V}{k T}\right]$

$A^{*}$ étant la constante de Richardson effective et $T$ la température. On néglige donc l'influence des phénomènes de diffusion (importants lorsque le libre parcours moyen $l$ est inférieur à $W$ ) et d'effet tunnel sur la probabilité de transfert d'un électron à travers la barrière. Les caractéristiques en régime de diffusion ont été calculées mais elles ne sont pas mentionnées ici en raison de leur similitude avec les résultats qui sont dérivés de la relation (5). L'influence de l'effet tunnel sera réexaminée dans la discussion des résultats.

2.4 COMPORTEMENT AU-DESSUS DE $V_{\mathrm{s}}$ (Fig. 1b). Au paragraphe suivant, on calcule le taux de génération $G$, rapport entre le courant de trous $J_{t}$ et le courant des électrons primaires ayant franchi la barrière. Pour les polarisations voisines de $V_{s}$, ce dernier est égal à $\left(J_{\mathrm{e}}-J_{\mathrm{t}}\right)$ puisque le courant d'émission thermoionique à partir du grain polarisé positivement est alors négligeable. On fait de plus l'approximation $J_{\mathrm{e}}-J_{\mathrm{t}} \simeq J_{\mathrm{e}}$, valable tant que $G \ll 1$, ce qui est le cas de tous les résultats présentés au paragraphe 3. C'est donc finalement la relation simple $J_{\mathrm{t}}=G J_{\mathrm{e}}$ qui est utilisée.

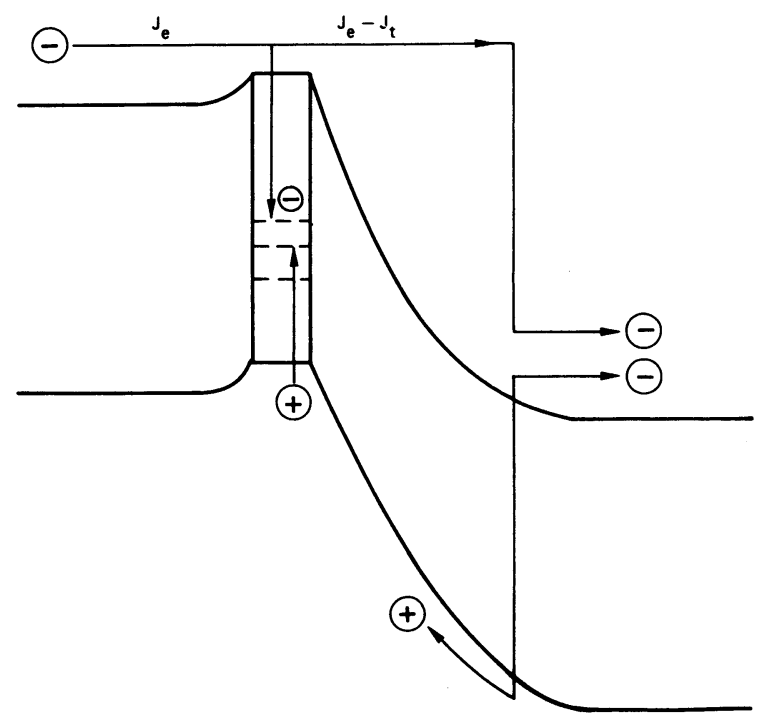

Fig. 1b. - Courants et processus de création et de recombinaison de trous considérés dans le modèle, dans le cas d'une polarisation $V>V_{\mathrm{s}}$.]

[Currents and hole generating and recombining processes considered in the model, in the case of a polarization $V>V_{\text {s. }}$

Il faut maintenant calculer l'influence du courant $J_{\mathrm{t}}$ sur la charge $Q$. D'après les hypothèses faites en 2.1 , les trous collectés à l'interface sont piégés en totalié par les états localisés, puis se recombinent avec les électrons de la bande de conduction. Le taux de recombinaison associé à ce dernier processus est :

$$
1 / \tau_{\mathrm{t}}=n_{0} \sigma_{\mathrm{t}} v_{\mathrm{th}} / 2
$$

où $n_{0}$ est la densité électronique que l'on aurait au sommet d'une barrière de hauteur $\phi_{\mathrm{B}}$ symétrique (pour $V \gg k T$ la densité est égale à $n_{0} / 2$ ) :

$$
n_{0}=N_{\mathrm{c}} \exp -\frac{\phi_{\mathrm{B}}+\xi}{k T}
$$

Pour calculer la charge $Q$ correspondant à ce régime, il faut vérifier que la perturbation de l'interface par le courant $J_{\mathrm{t}}$ se traduit par une différence de charge $\Delta Q$ par rapport au cas où $J_{\mathrm{t}}=0$, avec :

$$
\Delta Q=-J_{\mathrm{t}} \tau_{\mathrm{t}}
$$

Remarquons que ceci n'est pas évident a priori dans la mesure où la perturbation de charge se répercute sur les courants de capture et d'émission des électrons de conduction par les pièges de l'interface (ainsi la relation (8) avec $\tau$ défini par (6) serait fausse dans le cas d'un équilibre thermique entre les pièges de l'interface). Dans le cadre des hypothèses du modèle, la fonction de distribution d'occupation $f\left(E_{\mathrm{t}}\right)$ des pièges est donnée par :

$$
f\left(E_{\mathrm{t}}\right)=c /\left(c+e+e^{\prime}\right)
$$

où $c, e$ et $e^{\prime}$ sont définis de la façon suivante : le taux de capture $c$ des électrons de conduction est égal à $1 / \tau_{t}$. La section efficace $\sigma_{t}^{\prime}$ étant supposée indépendante de l'énergie, il en est de même pour le taux de capture $e^{\prime}$ des trous constituant le courant $J_{t}$. On a donc $e^{\prime}=J_{\mathrm{t}} /\left(q N_{\sigma \mathrm{c}}\right)$ où $N_{\sigma \mathrm{c}}$ est la densité des pièges occupés par des électrons. Le taux d'émission $e$ des électrons par ceux-ci est :

$$
e=N_{\mathrm{c}} \sigma_{\mathrm{t}} v_{\mathrm{th}} \exp -\frac{E_{\mathrm{g}}-E_{\mathrm{t}}}{k T}
$$

La variation de charge $\Delta Q$ est donnée par :

$$
\Delta Q=q N_{\mathrm{i}} \int_{0}^{E_{\mathrm{g}}}\left[f\left(E_{\mathrm{t}}\right)-f_{0}\left(E_{\mathrm{t}}\right)\right] \mathrm{d} E_{\mathrm{t}}
$$

avec

$$
f_{0}\left(E_{\mathrm{t}}\right)=c /(c+e) \text {. }
$$

La comparaison des ordres de grandeur de $c, e$ et $e^{\prime}$ montre que si $f\left(E_{t}\right)$ n'est plus comme 2.3 identique à une distribution Fermi-Dirac, la bande interdite reste partagée malgré tout en deux régions où $f\left(E_{\mathrm{t}}\right)$ est constant :

- Pour $E_{\mathrm{t}}>E_{\mathrm{g}}-\phi_{\mathrm{B}}-\xi$ on a : $e \gg c$, ce qui entraîne :

$$
f\left(E_{\mathrm{t}}\right)-f_{0}\left(E_{\mathrm{t}}\right) \simeq 0
$$


- Pour $E_{\mathrm{t}}<E_{\mathrm{g}}-\phi_{\mathrm{B}}-\xi$ on a $e \ll c ; e \ll e^{\prime}$ donc :

$$
f\left(E_{\mathrm{t}}\right)-f_{0}\left(E_{\mathrm{t}}\right) \simeq-e^{\prime} /\left(c+e^{\prime}\right) .
$$

Dans ces conditions, la relation (11) donne :

$$
\Delta Q=-q N_{\mathrm{i}}\left(E_{\mathrm{g}}-\phi_{\mathrm{B}}-\xi\right) \frac{J_{\mathrm{t}} \tau_{\mathrm{t}}}{q N_{\mathrm{oc}}+J_{\mathrm{c}} \tau_{\mathrm{t}}} .
$$

En remarquant que $-q N_{\mathrm{i}}\left(E_{\mathrm{g}}-\phi_{\mathrm{B}}-\xi\right)=q N_{\mathrm{oc}}-$ $\Delta Q$, on vérifie immédiatement (8) à partir de la relation précédente. $\Delta Q$, qui apparaît comme uniquement constitué des trous piégés à l'interface, sera donc noté $-Q_{\mathrm{t}}$ dans la suite de ce travail. La charge globale $Q$ devient alors :

$$
Q=Q_{0}+N_{\mathrm{i}}\left(\phi_{\mathrm{B}_{0}}-\phi_{\mathrm{B}}\right)-J_{\mathrm{t}} \tau_{\mathrm{t}}
$$

\subsection{CAlCUl DU TAUX DE GÉNÉRATION DES TROUS.} - Les électrons ayant franchi la barrière par émission thermoionique sont fortement accélérés par le champ électrique qui règne dans la zone désertée de largeur $W_{2}$. Soit $E_{\mathrm{i}}$ le seuil d'énergie nécessaire pour créer une paire électron-trou par ionisation d'impact. La condition générale $E_{\mathrm{i}} \geqq E_{\mathrm{g}}$ indique que lorsque $q V \simeq E_{\mathrm{g}}$ les électrons doivent parcourir une distance $W_{\mathrm{i}}$ presque égale à $W_{2}$ avant d'acquérir l'énergie $E_{\mathrm{i}}$ (Fig. 2). La création de paires électron-trous ne peut donc intervenir que sur une fraction réduite de la zone désertée en question. Plus précisément, $W_{\mathrm{i}}$ est donné par :

$$
W_{\mathrm{i}}=W_{2}\left[1-\left(1-E_{\mathrm{i}} / q V_{2}\right)^{1 / 2}\right]
$$

avec :

$$
V_{2}=V+\phi_{\mathrm{B}} / q \text { et } W_{2}=\left[2 \varepsilon V_{2} / q N_{\mathrm{d}}\right]^{1 / 2}
$$

Dans cette situation, l'utilisation de la théorie des coefficients d'ionisation serait peu indiquée car les corrections à introduire en raison de la couche morte de largeur $W_{\mathrm{i}}$ seraient trop importantes. Elles seraient par ailleurs d'autant plus complexes que le champ n'est pas uniforme dans la région considérée. Le calcul du taux de génération des paires a donc été effectué par une méthode de Monte Carlo permettant de s'affranchir de ces difficultés.

Les hypothèses du calcul peuvent être énumérées comme suit :

- Les collisions électron-électron sont négligées en raison de la faible densité électronique dans la zone considérée.

- Le libre parcours moyen des électrons est essentiellement limité par des collisions inélastiques avec des phonons de forte énergie $E_{\mathrm{R}}$ (du même ordre que celle des phonons optiques à $k=0$ ). Le libre parcours moyen associé à ce processus est désigné par $\lambda_{\mathrm{R}}$.

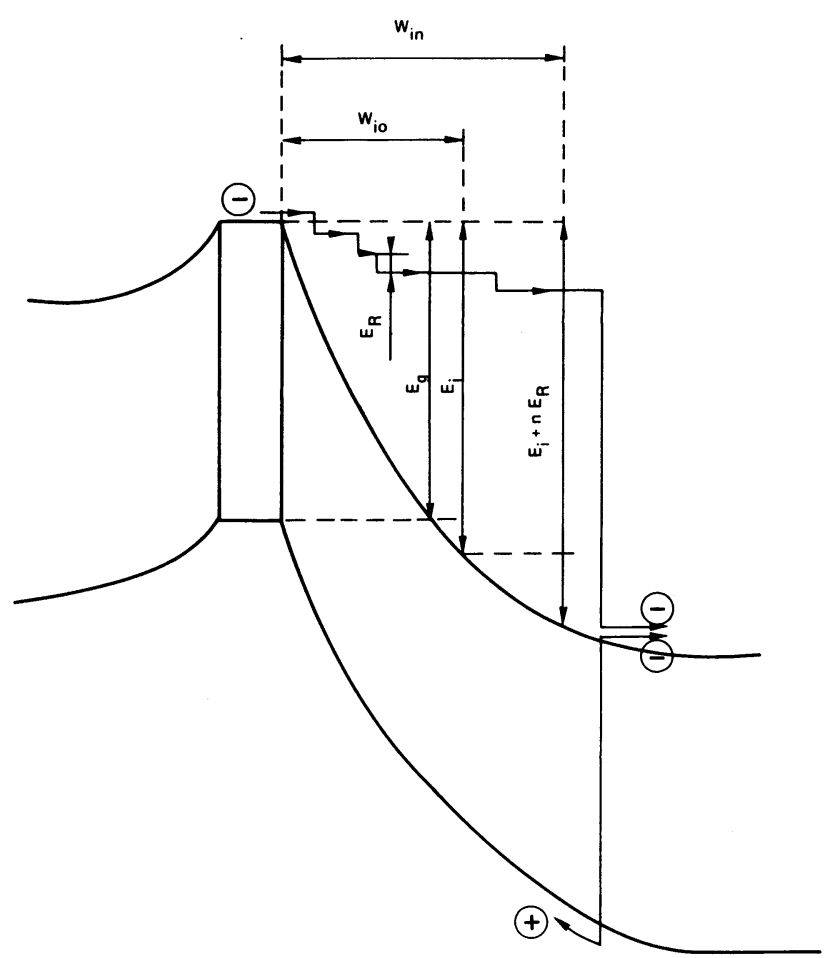

Fig. 2. - Schéma du processus d'ionisation d'impact après $n$ collisions électron-phonons ( $n=4$ dans l'exemple de trajectoire figuré).

[Diagram of the impact ionization process after $n$ electronphoton collisions $(n=4$ for the electron path represented as an example).]

- La section efficace de collision électron-phonon est supposée isotrope.

- $\lambda_{\mathrm{R}}$ est supposé indépendant de l'énergie cinétique des électrons, sauf naturellement lorsque celleci devient inférieure à $E_{\mathrm{R}}$ (on prend dans ce cas $\lambda_{\mathrm{R}}=\infty$ ).

- On néglige les processus d'absorption de phonons compte tenu du faible rapport $k T / E_{\mathrm{R}}$ à température ambiante.

- Le libre parcours moyen $\lambda_{\mathrm{i}}$ associé aux processus d'ionisation ne peut prendre que deux valeurs : $\lambda_{\mathrm{i}}=\infty$ si l'énergie cinétique des électrons est inférieure à $E_{\mathrm{i}} ; \lambda_{\mathrm{i}}=$ Cte dans le cas contraire. Les calculs de Baraff [16] montrent que le choix de ce paramètre n'est pas critique.

- On suppose que les électrons ont une énergie cinétique nulle lorsqu'ils franchissent la barrière.

Ces hypothèses correspondent à celles qui ont été habituellement retenues pour le calcul des coefficients d'ionisation. Un modèle plus complet ne semble pas nécessaire dans notre cas compte tenu de l'incertitude qui accompagne de toute façon le choix de $\lambda_{\mathrm{R}}$.

Les détails de la procédure de simulation sont donnés dans l'annexe 1. 
Le choix du paramètre $\lambda_{\mathrm{R}}$ (ou plus exactement du rapport $\lambda_{\mathrm{R}} / W_{2}$ ) contrôle de manière critique l'ordre de grandeur du taux de génération obtenu. Il n'existe pas à notre connaissance de détermination expérimentale de $\lambda_{\mathrm{R}}$ dans $\mathrm{ZnO}$. On a donc effectué les calculs pour différentes valeurs de ce paramètre. Celles-ci apparaissent vraisemblables compte tenu des valeurs observées pour $\lambda_{R}$ dans d'autres composés semiconducteurs [17].

Des résultats de la simulation Monte Carlo sont reportés sur la figure 3. Le taux de génération $G$ est calculé en fonction de la différence entre la chute de potentiel électrique $q V_{2}$ et l'énergie d'ionisation $E_{\mathrm{i}}$.

Le calcul fait intervenir la masse effective, des

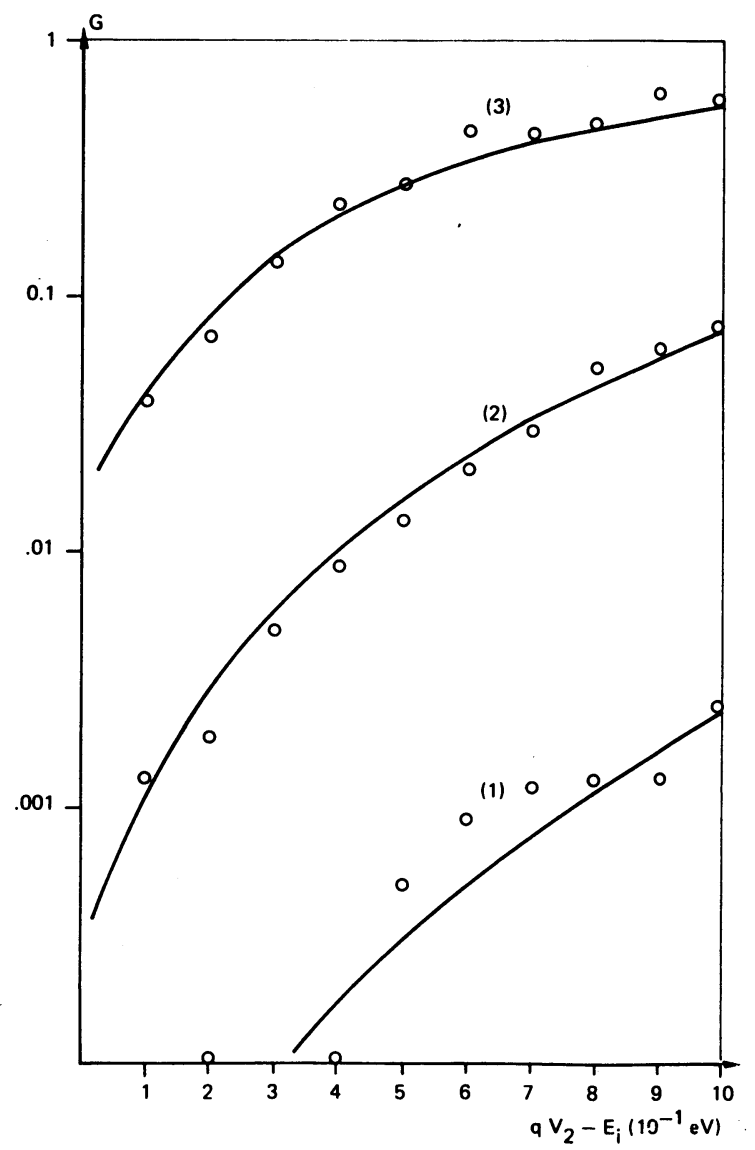

Fig. 3. - 0 : Simulation Monte Carlo du taux de génération $G$ des paires électron-trous :
(1) $\lambda_{\mathrm{R}}=3 \mathrm{~nm}$;
(2) $\lambda_{\mathrm{R}}=5 \mathrm{~nm}$;
(3) $\lambda_{\mathrm{R}}=10 \mathrm{~nm}$.

Les courbes représentent les variations de la relation empirique (21) pour les valeurs correspondantes de $\lambda_{\mathrm{R}}$.

[O : Monte Carlo simulation of the generation rate $G$ for electron-hole pairs :
(1) $\lambda_{\mathrm{R}}=3 \mathrm{~nm}$;
(2) $\lambda_{\mathrm{R}}=5 \mathrm{~nm}$;
(3) $\lambda_{\mathrm{R}}=10 \mathrm{~nm}$.

The curves represent the variation of the empirical relation (21) for the corresponding values of $\lambda_{R}$.] électrons de conduction $m_{\mathrm{e}}^{*}=0,27 m_{\mathrm{e}}$, l'énergie des phonons $E_{\mathrm{R}}=0,0722 \mathrm{eV}$ et la constante diélectrique $\varepsilon$ de $\mathrm{ZnO}\left(\varepsilon=8,5 \varepsilon_{0}\right)$.

Dans les résultats présentés sur la figure 3, $N_{\mathrm{d}}$ a été pris égal à $2,5 \times 10^{18} / \mathrm{cm}^{3}$. (Un balayage sur différentes valeurs de ce paramètre est effectué au paragraphe suivant.)

La validité de la simulation Monte Carlo a été contrôlée en comparant ces résultats avec des formules analytiques qu'il est possible d'obtenir moyennant une modification des hypothèses précédemment exposées. Deux cas ont été examinés à cet égard :

- Dans le premier cas, on suppose que tout électron subissant une collision avec un phonon perd la possibilité de provoquer ultérieurement la création d'une paire électron-trou. C'est l'hypothèse de départ adoptée par Schockley pour l'estimation des coefficients d'ionisation [18]. Dans ce cas, le taux de génération $G$ est donné par la probabilité pour un électron de ne pas subir de collision sur la distance $W_{\text {io }}$ :

$$
W_{\mathrm{io}}=W_{2}\left[\left(1-E_{\mathrm{R}} / q V_{2}\right)^{1 / 2}-\left(1-E_{\mathrm{i}} / q V_{2}\right)^{1 / 2}\right]
$$

et de subir ensuite un processus d'ionisation d'impact ; on a alors :

$$
G=\exp \left(-\frac{W_{\text {io }}}{\lambda_{\mathrm{R}}}\right) \frac{\lambda_{\mathrm{R}}}{\lambda_{\mathrm{i}}+\lambda_{\mathrm{R}}} .
$$

On obtient ainsi un minorant du taux de génération réel.

- Dans le deuxième cas, on suppose que les collisions électron-phonon ne perturbent que le module et non l'orientation de la vitesse des électrons. Le calcul analytique correspondant est présenté dans l'annexe 2. Le taux de génération peut être exprimé par :

$$
G=\sum_{N=0}^{N_{\max }} P_{\mathrm{i} N}\left[1-\left(\frac{\lambda_{\mathrm{i}}}{\lambda_{\mathrm{i}}+\lambda_{\mathrm{R}}}\right)^{N_{\max }-N+1}\right]
$$

$N_{\text {max }}$ étant la partie entière de $\left(q V_{2}-E_{\mathrm{i}}\right) / E_{\mathrm{R}}$ et $P_{\mathrm{i} N}$ étant donné par :

$$
\begin{aligned}
& P_{\mathrm{i} N}=\exp \left(-\frac{W_{\mathrm{i} N}}{\lambda_{\mathrm{R}}}\right)\left[\frac{1}{N !}\left(\frac{W_{\mathrm{i} N}}{\lambda_{\mathrm{R}}}\right)^{N}-\right. \\
& \left.-\sum_{n=0}^{N-1} \frac{1}{n !}\left(\frac{W_{\mathrm{i} n}}{\lambda_{\mathrm{R}}}\right)^{n} \frac{1}{(N-n) !}\left(\frac{W_{\mathrm{i} N}-W_{\mathrm{i} n}}{\lambda_{\mathrm{R}}}\right)^{N-n}\right] .
\end{aligned}
$$

Dans cette expression, $W_{\text {in }}$ est la distance que doit parcourir un électron d'énergie $E_{\mathrm{R}}$ pour acquérir l'énergie $E_{\mathrm{i}}$ tout en subissant $n$ collisions (Fig. 2) :

$$
W_{\mathrm{i} n}=W_{2}\left[\left(1-\frac{E_{\mathrm{R}}}{q V_{2}}\right)^{1 / 2}-\left(1-\frac{E_{\mathrm{i}}+n E_{\mathrm{R}}}{q V_{2}}\right)^{1 / 2}\right] \text {. }
$$


Ces relations sont justifiées dans l'annexe 2. Les distances à parcourir par les électrons avant de pourvoir donner lieu à une ionisation d'impact sont alors minimales puisque les composantes parallèles à l'interface du mouvement sont négligées. Ceci réduit la probabilité d'occurrence du processus concurrent de collision électron-phonon. La relation (18) correspond donc à un majorant de $G$.

Les résultats analytiques (17) et (18) et leurs équivalents en simulation Monte Carlo sont représentés sur la figure 4 . On peut vérifier par comparaison avec la figure 3 que les relations (17) et (18) jouent bien respectivement le rôle d'un minorant et d'un majorant. Par ailleurs, les résultats de simulation Monte Carlo recoupent les résultats analytiques

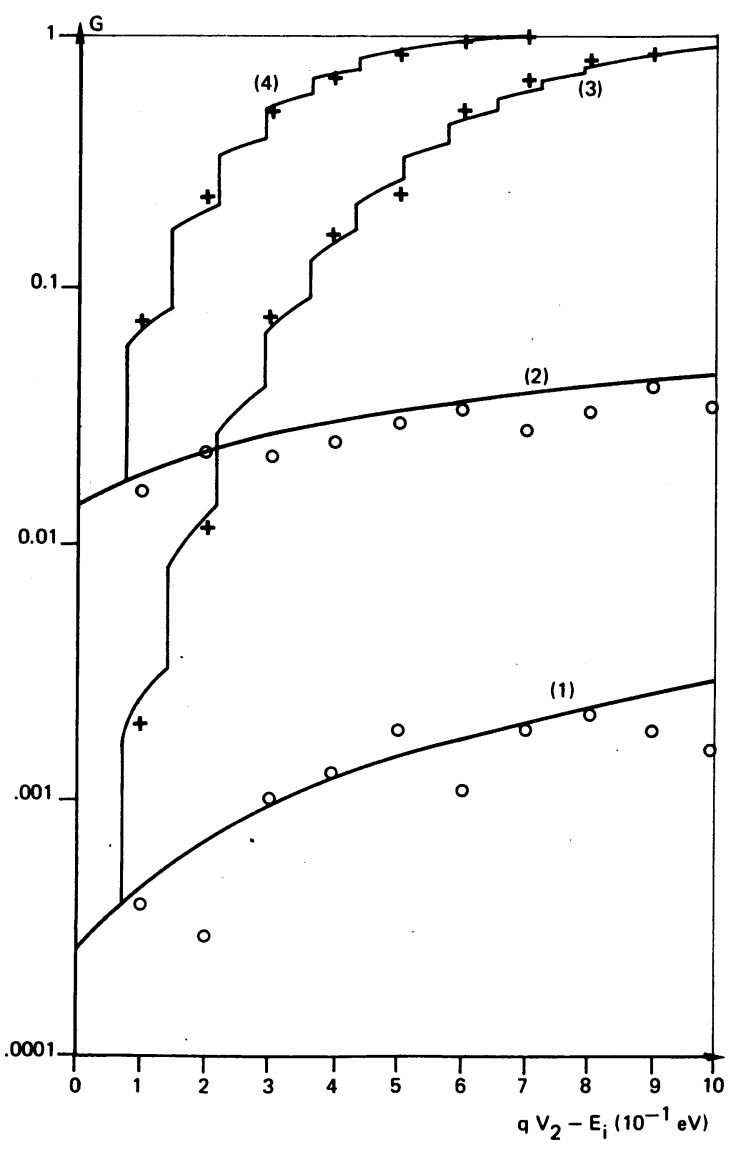

Fig. 4. - Tests de validité de la simulation Monte Carlo. $O$ : Simulation Monte Carlo des minorants analytiques pour $\lambda_{\mathrm{R}}=5 \mathrm{~nm}(1)$ et $\lambda_{\mathrm{R}}=10 \mathrm{~nm}(2) .+$ : Simulation Monte Carlo des majorants analytiques pour $\lambda_{R}=$ $5 \mathrm{~nm}$ (3) et $\lambda_{\mathrm{R}}=10 \mathrm{~nm}$ (4). Les courbes tracées représentent les expressions analytiques (17) et (18) calculées pour les valeurs de $\lambda_{R}$ correspondantes.

[Monte Carlo simulation validity test. $\mathrm{O}$ : Monte Carlo simulation of analytic lower bounds for $\lambda_{R}=5 \mathrm{~nm}$ (1) and $\lambda_{\mathrm{R}}=10 \mathrm{~nm}(2) .+$ : Monte Carlo simulation of the analytic upper bounds for $\lambda_{\mathrm{R}}=5 \mathrm{~nm}$ (3) and $\lambda_{\mathrm{R}}=10 \mathrm{~nm}$ (4). The plotted curves represent the analytic expressions (17) and (18) calculated for the corresponding values of $\lambda_{\mathrm{R}}$.] avec une précision convenable compte tenu des quelques sources d'erreurs inhérentes au calcul (fluctuations statistiques, valeur finie du pas de temps, etc...).

La simulation Monte Carlo permet de prendre en compte des hypothèses plus réalistes que les approximations apalytiques (17) et (18) mais elle est plus lourde d'emploi. Cet inconvénient a pu être éliminé en constatant que ses résultats sont reproduits avec une excellente précision par la relation empirique :

$$
G=x /(1+x)
$$

avec :

$$
\begin{aligned}
x=\exp \left(-\frac{W_{\text {io }}}{\lambda_{\mathrm{R}}}\right) & \left(\frac{\lambda_{\mathrm{R}}}{\lambda_{\mathrm{i}}+\lambda_{\mathrm{R}}}\right) \times \\
\times & {\left[1+0,82\left(\frac{q V_{2}-E_{\mathrm{i}}}{E_{\mathrm{R}}}\right)^{1,3}\right] . }
\end{aligned}
$$

La relation (21) est comparée sur la figure 3 aux résultats de la simulation Monte Carlo.

On a vérifié que la précision de l'accord obtenu ne dépend pas de $N_{\mathrm{d}}$ (tout au moins dans le domaine de paramétrage utilisé dans la section 3 ). Ce sont donc les relations (21) et (22) qui ont été utilisées dans les résultats présentés ci-dessous.

2.6 INFLUENCE DE LA RÉSISTANCE VOLUMIQUE DES GRAINS. - On la prend en considération en ajoutant à la tension $V$ un terme $\rho d J_{\mathrm{e}}$ où $\rho$ est la résistivité de l'oxyde de zinc dans les régions neutres, $d$ est le diamètre effectif des grains (tenant compte du fait que certains d'entre eux sont en court-circuit avec un ou plusieurs grains voisins). Le champ électrique macroscopique $F$ dans la varistance est alors :

$$
F=\frac{V}{d}+\rho J_{\mathrm{e}}
$$

2.7 CHOIX DES DIFFÉRENTS PARAMÈTRES. - Les paramètres physiques qui entrent dans le modèle peuvent être répartis en trois catégories :

2.7.1 Grandeurs connues avec une bonne précision. - La permittivité $\varepsilon$ est égale à $8,5 \varepsilon_{0}$ ( $\varepsilon_{0}$ étant la constante diélectrique du vide) ; la masse effective $m_{\mathrm{e}}^{*}$ des électrons de conduction est égale à $0,27 m_{\mathrm{e}}$ ( $m_{\mathrm{e}}$ étant la masse de l'électron libre); l'énergie des phonons peut être prise égale à $0,072 \mathrm{eV}$ (voir paragraphe précédent). On peut déduire de $m_{\mathrm{e}}^{*}$ :

$$
\begin{gathered}
A^{*} \simeq 30 \mathrm{~A} / \mathrm{cm}^{2} ; \\
v_{\text {th }}=\left(8 k T / \pi m_{\mathrm{e}}^{*}\right)^{1 / 2}=2,1 \times 10^{7} \mathrm{~cm} / \mathrm{s}
\end{gathered}
$$

et

$$
N_{\mathrm{c}}=3,5 \times 10^{18} / \mathrm{cm}^{3}(\mathrm{à} 300 \mathrm{~K}) .
$$

2.7.2 Grandeurs faisant l'objet de déterminations expérimentales approximatives. - Certaines mesures effectuées sur les varistances donnent des indications 
sur la valeur des paramètres $\phi_{\mathrm{B}_{0}}, \xi, \rho$ et $d$. Des mesures récentes de conductivité à haute fréquence ont montré que la résistivité volumique des grains variait entre 2 et $3 \Omega . c m$ en fonction de la teneur en impuretés [19]. Sachant que la mobilité (à faible champ) des porteurs dans l'oxyde de zinc dopé à ces niveaux est généralement de l'ordre de $10^{2} \mathrm{~cm}^{2} / V$.s, on en déduit une concentration électronique $n$ de l'ordre de $2,5 \times 10^{16} \mathrm{~cm}^{-3}$ dans les régions neutres. On peut alors évaluer $\xi$ par :

$$
\xi=k T \log \frac{N_{\mathrm{c}}}{n} \simeq 0,13 \mathrm{eV} .
$$

Ce paramètre ne jouant pas un rôle primordial dans le modèle, aucun balayage autour de cette valeur n'a été effectué. La hauteur de barrière $\phi_{\mathrm{B} 0}$ peut être estimée d'après l'énergie d'activation $E_{\mathrm{a}}$ de la conductivité sous faible polarisation, en général voisine de $0,8 \mathrm{eV} . E_{\mathrm{a}}$ donne accès à $\phi_{\mathrm{B}_{0}}+\xi$ moyennant une correction liée à $\partial \phi_{\mathrm{B}_{0}} / \mathrm{d} T$ qui est mal connu. On a donc effectué un balayage sur différentes valeurs de $\phi_{\mathrm{B}_{0}}$ comprises entre 0,7 et $1 \mathrm{eV}$, intervalle de variation considéré comme vraisemblable. Le diamètre des grains $d$ a été pris égal à $20 \mu \mathrm{m}$, valeur conforme aux résultats d'examen de la microstructure des varistances [20]. Un choix différent amènerait une homothétie en tension des caractéristiques $F\left(J_{\mathrm{e}}\right)$. Cette opération n'aurait pas grande signification dans la mesure où, l'énergie d'ionisation $E_{\mathrm{i}}$ étant mal connue, le rapport $E_{\mathrm{i}} / d$ doit de toute façon être considéré comme un paramètre ajustable permettant de retrouver une valeur correcte pour le champ caractéristique de la zone II. Pour $d=20 \mu \mathrm{m}$, ceci conduit à $E_{\mathrm{i}} \simeq 4 \mathrm{eV}$.

2.7.3 Grandeurs mal connues. - Les mesures de capacité ont conduit à des estimations de $N_{\mathrm{d}}$ généralement comprises entre quelques $10^{17}$ et $10^{19}$ $[19,21,22]$, intervalle de variation qui est reproduit dans les simulations présentées ci-dessous. L'ordre de grandeur de $N_{\mathrm{i}}$ est estimé en considérant que l'interface intergranulaire est vraisemblablement une zone fortement perturbée dans laquelle une fraction notable $(\simeq 10 \%)$ des sites atomiques présentent un état localisé. Dans la mesure où ces états sont répartis à peu près uniformément dans la bande interdite, ceci correspondrait à une densité de quelques $10^{13}\left(\mathrm{~cm}^{2} \mathrm{eV}\right)^{-1}$. On a donc choisi de faire varier $N_{\mathrm{i}}$ entre $10^{13}$ et $10^{14}\left(\mathrm{~cm}^{2} \mathrm{eV}\right)^{-1}$. En ce qui concerne la section efficace de capture $\sigma_{\mathfrak{t}}$, l'intervalle de variation choisi pour les modélisation a pour limites $3 \times 10^{-14}$ et $3 \times 10^{-16} \mathrm{~cm}^{2}$, ce qui correspond à des ordres de grandeur couramment observés.

Le libre parcours moyen $\lambda_{i}$, qui ne joue pas un rôle critique, est pris égal à $10 \mathrm{~nm}$. Au contraire, le modèle est très sensible au choix de $\lambda_{R}$, qu'on ne peut malheureusement estimer que par analogie avec des résusltats expérimentaux obtenus sur d'autres matériaux semi-conducteurs. Ces résultats indiquent une valeur de $\lambda_{\mathrm{R}}$ égale à quelques $\mathrm{nm}$ [17]. Dans les modélisations présentées ci-dessous, on a fait varier $\lambda_{\mathrm{R}}$ de 2,5 à $7,5 \mathrm{~nm}$.

Afin de limiter le nombre des caractéristiques modélisées, on a choisi de ne faire varier qu'un seul paramètre à la fois, les autres prenant alors une valeur standard, définie dans le tableau I où dans le texte précédent.

Tableau I. - Domaine de paramétrage étudié.

[Variations range considered for some parameters].

\begin{tabular}{|c|c|c|}
\hline Paramètre & Valeur standard & $\begin{array}{c}\text { Intervalle de } \\
\text { variation étudié }\end{array}$ \\
\hline$\phi_{\mathrm{B}_{0}}$ & $0,85 \mathrm{eV}$ & $0,72 \mathrm{à} 0,98 \mathrm{eV}$ \\
$N_{\mathrm{d}}$ & $2,5 \times 10^{18} \mathrm{~cm}^{-3}$ & $3 \times 10^{17} \mathrm{a} 10^{19} \mathrm{~cm}^{-3}$ \\
$N_{\mathrm{i}}$ & $2 \times 10^{13} \mathrm{~cm}^{-2} \mathrm{eV}^{-1}$ & $10^{13} \mathrm{à} 10^{14} \mathrm{~cm}^{-2} \mathrm{eV}^{-1}$ \\
$\sigma_{\mathrm{t}}$ & $10^{-15} \mathrm{~cm}^{2}$ & $3 \times 10^{-14} \mathrm{à} 3 \times 10^{-16} \mathrm{~cm}^{2}$ \\
$\lambda_{\mathrm{R}}$ & $4 \mathrm{~nm}$ & $2,5 \mathrm{à} 7,5 \mathrm{~nm}$ \\
$\rho$ & $2,5 \Omega \cdot \mathrm{cm}$ & - \\
\hline
\end{tabular}

\section{Présentation et discussion des résultats.}

Le système d'équations développé dans la section 2 est résolu numériquement au moyen d'un programme de calcul utilisant une méthode d'approximations successives.

On présente ci-dessous trois groupes de résultats. Le premier a pour but de montrer de façon explicite le comportement des principales grandeurs physiques qui caractérisent la DBS, à savoir les hauteurs de barrière $\phi_{\mathrm{B}}$ et $q V_{2}$, ainsi que les composantes $Q_{\mathrm{e}}$ et $Q_{\mathrm{t}}$ de la charge d'interface. Le second effectue une comparaison avec une caractéristique $F\left(J_{\mathrm{e}}\right)$ expérimentale afin de démontrer l'aptitude du modèle à reproduire celle-ci. Le dernier groupe de résultats est destiné à établir que l'allure générale des caractéristiques modélisées est conservée dans tout le domaine de paramétrage défini à la fin de la section 2 .

\subsection{COMPORTEMENT DES HAUTEURS DE BARRIÈRES} ET DES CHARGES D'INTERFACE. - Il est représenté sur la figure 5 en fonction de la densité de courant $J_{\mathrm{e}}$. On a affecté aux divers paramètres leur valeur standard. Les différences $V_{2}-\phi_{\mathrm{B}} / q$ et $Q_{\mathrm{e}}-Q_{\mathrm{t}}$ permettent d'obtenir respectivement la polarisation $V$ et la densité $Q$ de charge nette piégée à l'interface. On observe un comportement linéaire de $\phi_{\mathrm{B}} / q$ et de $Q_{\mathrm{e}}$ en fonction de $\log \left(J_{\mathrm{e}}\right)$, qui résulte de la nature des équations utilisées.

Pour $q V<E_{\mathrm{i}}$, c'est essentiellement sur la tension $V_{2}$ que se reportent les variations de polarisation. L'apparition de la composante $Q_{\mathrm{t}}$ de la charge 


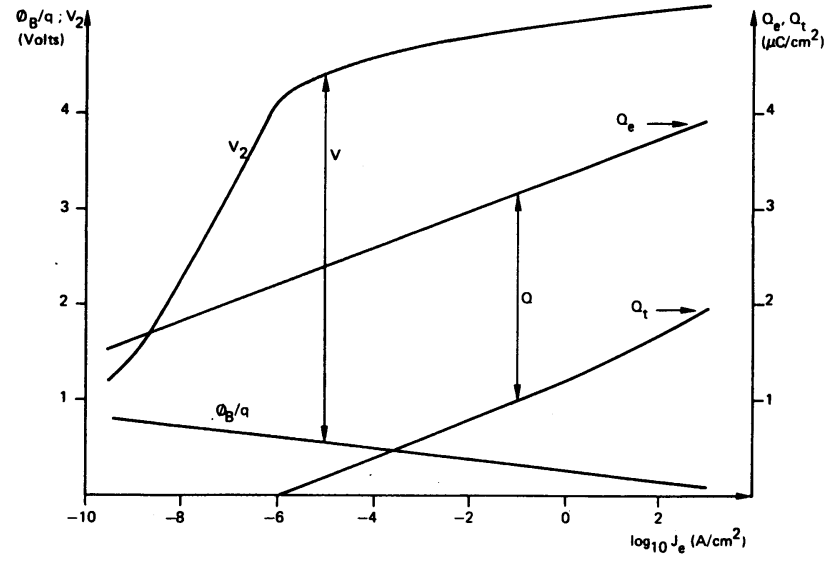

Fig. 5. - Comportement de la DBS en fonction de la densité de courant $J_{\mathrm{e}}$. La polarisation $V$ est donnée par la différence $V_{2}-\phi_{\mathrm{B}} / q$ et la densité de charge nette piégée à l'interface $Q$ par la différence $Q_{\mathrm{e}}-Q_{\mathrm{t}}$.

[DBS behaviour as a function of current density $J_{\mathrm{e}}$. The polarization, $V$, is given by the difference $V_{2}-\phi_{\mathrm{B}} / q$ and the net charge density trapped at the interface $Q$ is given by the difference $Q_{\mathrm{e}}-Q_{\mathrm{t}}$.]

d'interface fixe $Q$ à une valeur pratiquement constante pour $q V>E_{\mathrm{i}}$. Les variations de $V$ se répartissent alors de façon à peu près équivalente entre $\phi_{\mathrm{B}} / q$ et $V_{2}$. Ceci se traduit par un accroissement brutal de la non-linéarité de $J_{\mathrm{e}}(V)$ puisque la densité de courant est contrôlée par $\phi_{\mathrm{B}}$.

3.2 SimULATION D'UNE CARACTÉRISTIQUE $F\left(J_{\mathrm{e}}\right)$ EXPÉRIMENTALE. - Les caractéristiques électriques des varistances provenant des divers constructeurs présentent des différences quantitatives importantes, en particulier sur la tension de seuil, alors que leur allure reste qualitativement identique. Ceci traduit vraisemblablement la permanence des mécanismes physiques de base, alors que certains paramètres contrôlant la caractéristique $\left(d, \phi_{\mathrm{B}_{0}}, N_{\mathrm{d}}, N_{\mathrm{i}}\right.$, $\rho)$ varient en fonction du processus de fabrication. Il est donc légitime de rechercher un ajustement optimal de ces paramètres lorsque l'on tente de reproduire une caractéristique expérimentale au moyen du modèle.

A titre d'exemple, on a reporté sur la figure 6 une caractéristique mesurée (extraite de la Réf. [23]) et une caractéristique calculée par le présent modèle avec le choix de paramètres suivants: $\phi_{\mathrm{B}_{0}}=$ $0,84 \mathrm{eV} ; \quad d=18,7 \mu \mathrm{m} ; \quad \sigma_{\mathrm{t}}=2,5 \times 10^{-15} \mathrm{~cm}^{2}$; $\lambda_{\mathrm{R}}=5 \mathrm{~nm}$. Les autres paramètres ont leur valeur standard. Les coefficients de non-linéarité sont également reportés sur la figure 6. L'accord obtenu est satisfaisant sauf dans le domaine des forts courants. Etant donné que l'amélioration de l'ajustement nécessiterait un choix de la résistivité volumique $\rho$ non conforme aux résultats cités dans la section précédente, l'écart constaté a vraisemblablement

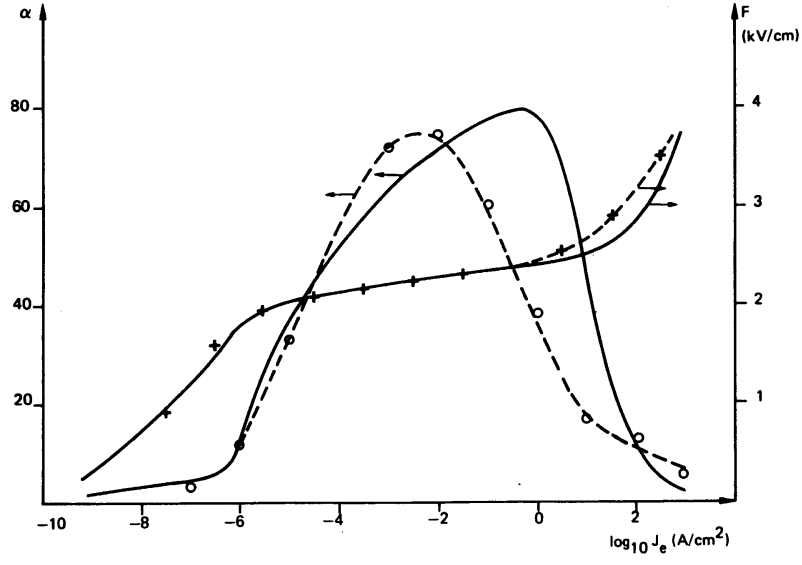

Fig. 6. - Caractéristiques du champ électrique et du coefficient de non-linéarité en fonction de la densité de courant. Les courbes en trait plein représentent les résultats du modèle. Les résultats expérimentaux [23] sont figurés en pointillés.

[Characteristics of the electric field and of the nonlinearity coefficient as a function of current density. Full line : results of the model. Dotted lines : experimental results [23].]

une autre cause. On peut remarquer que la caractéristique expérimentale à fort courant a été obtenue en régime impulsionnel. Il est bien connu que dans ce cas la tension crête mesurée est plus élevée que la tension continue équivalente [24]. Une extension du modèle au régime impulsionnel est en cours pour rendre compte de cet effet. Cependant, il existe plusieurs raisons pour lesquelles le modèle devient de toute façon moins précis en courant élevé. Tout d'abord, on ne peut plus supposer $\phi_{\mathrm{B}} \gg \xi \mathrm{ni}$ $W_{1} \gg L_{\mathrm{D}}\left(L_{\mathrm{D}}\right.$ étant la longueur de Debye $)$. Les hypothèses $\rho(x)=0$ pour $x<-W_{1}$ et $\rho(x)=$ $q N_{\mathrm{d}}$ pour $x>-W_{1}$ deviennent donc critiquables. Par ailleurs, des écarts apparaissent au comportement thermoionique simple exprimé par la relation (5) pour les faibles valeurs de $\phi_{\mathrm{B}}$. On peut enfin remarquer que lorsque $E_{\mathrm{g}}-E_{\mathrm{Fi}} \rightarrow 0$, la probabilité d'émission par effet de champ des électrons piégés sur les états d'interface peut devenir égale ou supérieure à la probabilité d'émission thermique et que dans ce cas l'approximation $E_{\mathrm{F} 1} \simeq E_{\mathrm{Fi}}(\S 2.3)$ n'est plus valable.

\subsection{SENSIBILITÉ DU MODÈlE AUX VARIATIONS DE} PARAMÈTRES. - En dehors des variations de paramètres prévues au paragraphe 2.7 , il convient de modéliser la caractéristique $F\left(J_{\mathrm{e}}\right)$ pour différentes valeurs de la température.

Les figures $7 \mathrm{a}$ à $7 \mathrm{f}$ donnent ces résultats en présentant à chaque fois la courbe de référence correspondant au jeu de paramètres standard et les caractéristiques obtenues pour les valeurs extrêmes données dans le tableau I. 

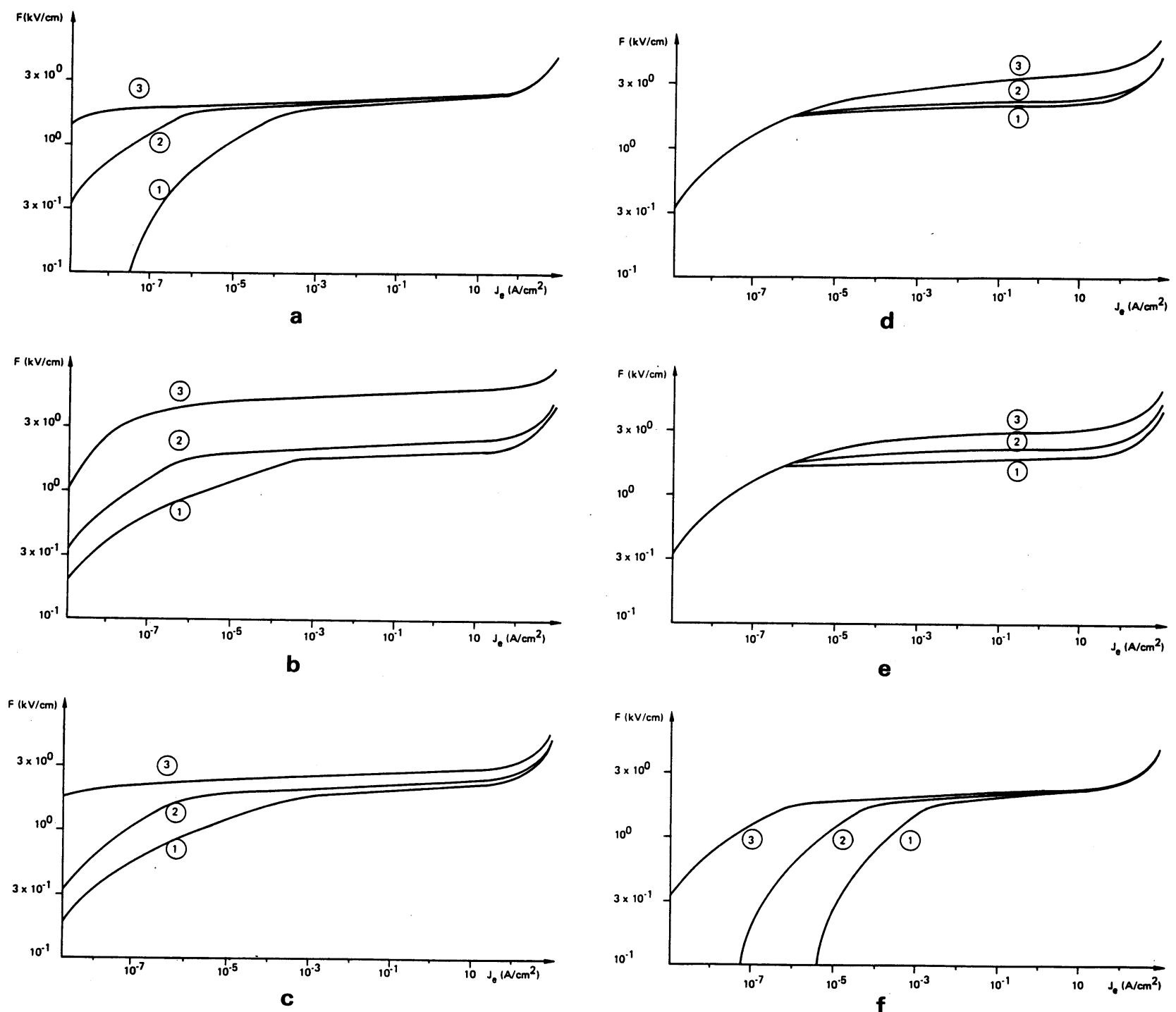

Fig. 7. Etude de la sensibilité des caractéristiques couranttension modélisées vis-à-vis des paramètres suivants : a) Hauteur de barrière : (1) $\phi_{\mathrm{B}_{0}}=0,72 \mathrm{eV}$; (2) $\phi_{\mathrm{B}_{0}}=$ $0,85 \mathrm{eV}$; (3) $\phi_{\mathrm{B}_{0}}=0,98 \mathrm{eV}$. b) Densité volumique des donneurs : (1) $N_{\mathrm{d}}=10^{19} \mathrm{~cm}^{-3}$; (2) $N_{\mathrm{d}}=2,5 \times$ $10^{18} \mathrm{~cm}^{-3}$; (3) $N_{\mathrm{d}}=3 \times 10^{17} \mathrm{~cm}^{-3}$. c) Densité d'états à l'interface : (1) $N_{\mathrm{i}}=10^{13} \mathrm{~cm}^{-2} \mathrm{eV}^{-1}$; (2) $N_{\mathrm{i}}=2 \times$ $10^{13} \mathrm{~cm}^{-2} \mathrm{eV}^{-1}$; (3) $N_{\mathrm{i}}=10^{14} \mathrm{~cm}^{-2} \mathrm{eV}^{-1}$.

Fig. 7. - d) Section efficace de recombinaison des trous : (1) $\sigma_{\mathrm{t}}=3 \times 10^{-16} \mathrm{~cm}^{2}$; (2) $\sigma_{\mathrm{t}}=10^{-15} \mathrm{~cm}^{2}$; (3) $\sigma_{\mathrm{t}}=$ $3 \times 10^{-14} \mathrm{~cm}^{2}$. e) Libre parcours moyen $\lambda_{\mathrm{R}}$ : (1) $\lambda_{\mathrm{R}}=$ $7,5 \mathrm{~nm}$; (2) $\lambda_{\mathrm{R}}=4 \mathrm{~nm}$; (3) $\lambda_{\mathrm{R}}=2,5 \mathrm{~nm}$. f) Température : (1) $T=400 \mathrm{~K}$; (2) $T=350 \mathrm{~K}$; (3) $T=300 \mathrm{~K}$.

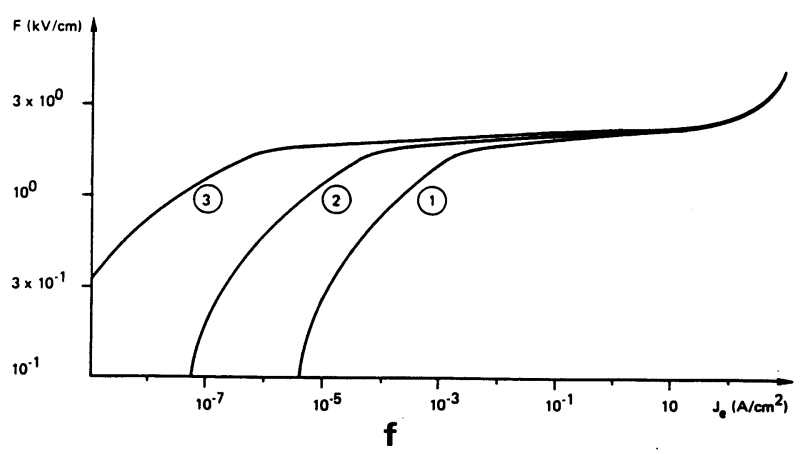

[Sensitivity of current-voltage characteristics to variations of the following parameters: a) Barrier height: (1) $\phi_{\mathrm{B}_{0}}=0.72 \mathrm{eV}$; (2) $\phi_{\mathrm{B}_{0}}=0.85 \mathrm{eV}$; (3) $\phi_{\mathrm{B}_{0}}=0.98 \mathrm{eV}$. b) Volumic donor density: (1) $N_{\mathrm{d}}=10^{19} \mathrm{~cm}^{-3}$; (2) $N_{\mathrm{d}}=2,5 \times 10^{18} \mathrm{~cm}^{-3}$; (3) $N_{\mathrm{d}}=3 \times 10^{17} \mathrm{~cm}^{-3}$. c) Density of states at the interface : (1) $N_{i}=10^{13} \mathrm{~cm}^{-2} \mathrm{eV}^{-1}$; (2) $N_{\mathrm{i}}=3 \times 10^{13} \mathrm{~cm}^{-2} \mathrm{eV}^{-1}$; (3) $N_{\mathrm{i}}=10^{14} \mathrm{~cm}^{-2} \mathrm{eV}^{-1}$.]

[d) Hole recombination cross section: (1) $\sigma_{\mathrm{t}}=3 \times$ $10^{-16} \mathrm{~cm}^{2}$; (2) $\sigma_{\mathrm{t}}=10^{-15} \mathrm{~cm}^{2}$; (3) $\sigma_{\mathrm{t}}=3 \times 10^{-14} \mathrm{~cm}^{2}$. e) Mean free path : (1) $\lambda_{\mathrm{R}}=7,5 \mathrm{~nm}$; (2) $\lambda_{\mathrm{R}}=4 \mathrm{~nm}$; (3) $\lambda_{\mathrm{R}}=2,5 \mathrm{~nm}$. f) Temperature : (1) $T=400 \mathrm{~K}$; (2) $T=$ $350 \mathrm{~K}$; (3) $T=300 \mathrm{~K}$.]

Il convient de remarquer que certaines combinaisons de choix de paramètres auraient pu conduire à des résultats très voisins les uns des autres. Ainsi, au-dessous de $V_{\mathrm{s}}$, la caractéristique $J_{\mathrm{e}}(V)$ ne dépend de $N_{\mathrm{d}}$ et $N_{\mathrm{i}}$ que par l'intermédaire du rapport $p_{1}=N_{\mathrm{i}}^{2} / N_{\mathrm{d}}$, [15]. Dans notre modèle, il apparaît que $G$ ne dépend que faiblement de $N_{\mathrm{d}}$ et $\lambda_{\mathrm{R}}$ dans la mesure où $p_{1}$ et le produit $p_{2}=\lambda_{\mathrm{R}} N_{\mathrm{d}}^{1 / 2}$ sont mainte- nus constants. Cependant chaque courbe de la figure 7 correspond à un couple $\left(p_{1}, p_{2}\right)$ particulier.

D'une façon générale les variations de paramètres effectuées conservent bien le comportement fortement non linéaire de la caractéristique $F\left(J_{\mathrm{e}}\right)$. La valeur maximale $\alpha_{\max }$ du coefficient de non-linéarité est donnée dans le tableau II. On constate que c'est seulement pour les plus fortes valeurs de $\sigma_{\mathfrak{t}}$ que 
Tableau II. - Valeurs maximales $\alpha_{\max } d u$ coefficient de non-linarité obtenues en faisant varier différents paramètres.

[Maximal values $\alpha_{\max }$ of the nonlinearity coefficient obtained from the variation of some parameters.]

\begin{tabular}{|c|c|c|c|c|c|c|c|}
\hline Fig. & Courbe & $\alpha_{\max }$ & Paramètre variant & Fig. & Courbe & $\alpha_{\max }$ & Paramètre variant \\
\hline $\begin{array}{l}\mathbf{a} \\
\mathbf{a}\end{array}$ & $\begin{array}{l}1 \\
3\end{array}$ & $\begin{array}{l}65 \\
93\end{array}$ & $\phi_{\mathrm{B}_{0}}$ & $\begin{array}{l}\mathrm{d} \\
\mathrm{d}\end{array}$ & $\begin{array}{l}1 \\
3\end{array}$ & $\begin{array}{l}92 \\
35\end{array}$ & $\sigma_{\mathrm{t}}$ \\
\hline $\begin{array}{l}\mathrm{b} \\
\mathrm{b}\end{array}$ & $\begin{array}{l}1 \\
3\end{array}$ & $\begin{array}{l}92 \\
87\end{array}$ & $N_{\mathrm{d}}$ & $\begin{array}{l}\mathrm{e} \\
\mathrm{e}\end{array}$ & $\begin{array}{l}1 \\
3\end{array}$ & $\begin{array}{r}109 \\
63\end{array}$ & $\lambda_{\mathrm{R}}$ \\
\hline c & 1 & $\begin{array}{l}64 \\
83\end{array}$ & $N_{\mathrm{i}}$ & $\begin{array}{l}\text { f } \\
\text { f } \\
\text { f }\end{array}$ & $\begin{array}{l}1 \\
2 \\
3\end{array}$ & $\begin{array}{ll}\text { (standard) } & 81 \\
62 \\
48\end{array}$ & $T$ \\
\hline
\end{tabular}

$\sigma_{\max }$ chute sensiblement au-dessous des valeurs expérimentales.

Les modélisations à température variable permettent de déduire un coefficient $\beta=d\left(\log F_{\mathrm{B}} / \mathrm{d} T\right)$ égal à $-10^{-3} \mathrm{~K}^{-1}, F_{\mathrm{B}}$ désignant le champ macroscopique obtenu pour $J_{\mathrm{e}}=10^{-2} \mathrm{~A} / \mathrm{cm}^{2}$. Sur les varistances $\mathrm{ZnO}$, on observe généralement $\beta=-2 \times$ $10^{-4} \mathrm{~K}^{-1}$ [25]. Cet écart peut être interprété en rappelant que $\lambda_{R}$ et $\sigma_{t}$ ont été supposés indépendants de $T$. Les variations naturellement attendues pour $\lambda_{\mathrm{R}}$ et $\sigma_{\mathrm{t}}\left(\mathrm{d} \lambda_{\mathrm{R}} / \mathrm{d} T<0\right.$ et $\left.\mathrm{d} \sigma_{\mathrm{t}} / \mathrm{d} T>0\right)$ conduisent toutes deux à une augmentation de la valeur algébrique calculée pour $\beta$, mais l'incertitude qui les caractérise rend pour l'instant illusoire une tentative de modélisation plus précise. En contrepartie le modèle actuel rend bien compte de l'évolution en température des caractéristiques $F\left(J_{\mathrm{e}}\right)$ dans la zone des courants inférieurs à $10^{-3} \mathrm{~A} / \mathrm{cm}^{2}$.

Les résultats présentés sur la figure $7 \mathrm{~b}$ montrent que $F_{\mathrm{B}}$ devient assez sensible au choix de $N_{\mathrm{d}}$ lorsque $N_{\mathrm{d}}<10^{18} \mathrm{~cm}^{-3}$. Les mesures effectuées sur les barrières intergranulaires individuelles montrent au contraire une dispersion assez faible de $V_{s}$ [11]. Certaines mesures de capacité conduisent effectivement à estimer que $N_{\mathrm{d}}$ pourrait être inférieur à $10^{18} \mathrm{~cm}^{-3}$, mais elles doivent être considérées avec prudence dans la mesure où les surfaces de contact intergranulaires sont mal connues, et où les effets dispersifs restent importants même à basse fréquence. Par ailleurs des concentrations de donneurs égales ou supérieures à $10^{18} \mathrm{~cm}^{-3}$ semblent vraisemblables compte tenu des fortes teneurs en impuretés utilisées lors de la fabrication des varistances.

\section{Comparaison avec d'autres mécanismes proposés.}

L'effet tunnel de type Zener peut en principe rendre compte de la non-linéarité des varistances avec une tension de seuil $V_{\mathrm{s}} \simeq E_{\mathrm{i}} / q$. Cependant, s'il s'agit d'effet tunnel direct (non assisté par des états présents dans la bande interdite) la largeur de zone désertée $W_{2}$ devrait être au plus de l'ordre de $10 \mathrm{~nm}$ lorsque $V \simeq V_{s}$, pour que la probabilité de transfert d'un élecron de la bande de valence à la bande de conduction soit significative. Ceci exige des valeurs de $N_{\mathrm{d}}$ encore plus élevées que celles que nous supposons ici.

L'effet tunnel assisté par des états localisés profonds est un mécanisme plausible [26], mais il apparaît particulièrement difficile à modéliser.

Il est important de remarquer que, bien qu'utilisant un processus de création de paires électrontrou, le présent modèle ne conduit pas à un comportement semblable à celui qui résulterait d'un phénomène d'avalanche. En particulier, le courant principal traversant l'interface étant d'origine thermoionique, le coefficient de température de $F_{B}$ peut être négatif. Par ailleurs, les tensions de claquage $V_{\mathrm{s}}$ que nous obtenons sont de l'ordre de $E_{\mathrm{g}} / q$ alors qu'un phénomène d'avalanche exige des tensions au moins deux à trois fois supérieures. Les arguments développés à l'encontre de celui-ci [27] ne s'appliquent donc pas au présent modèle.

\section{Conclusion.}

Les modélisations effectuées ont permis de montrer qu'un mécanisme d'ionisation d'impact est susceptible de rendre compte des fortes valeurs du coefficient de non-linéarité observées sur les varistances. Le modèle fait appel à un certain nombre d'hypothèses concernant les valeurs de quelques paramètres physiques qui ne sont pas bien connus expérimentalement. Cependant le comportement non linéaire est correctement restitué pour la quasi-totalité des choix de paramètres qui peuvent être considérés comme vraisemblables.

On constate que la valeur maximale du coefficient de non-linéarité est assez sensible aux valeurs du libre parcours moyen $\lambda_{R}$ relatif aux collisions entre 
électrons et phonons de forte énergie, et de la section efficace de recombinaison $\sigma_{\mathrm{t}}$ des trous piégés à l'interface. Par ailleurs le contrôle des paramètres $N_{\mathrm{d}}, N_{\mathrm{i}}$ et $\phi_{\mathrm{B}_{0}}$, qui devraient être assez dépendants des conditions de fabrication des varistances, semble nécessaire pour obtenir des caractéristiques tensioncourant reproductibles et présentant un coefficient de non-linéarité optimal.

\section{Annexe I. - Simulation par une méthode de Monte Carlo de la création des paires électron-trou par ionisation d'impact.}

Les électrons sont considérés comme des particules classiques. Leurs trajectoires sont repérées en coordonnées cylindriques $\left(^{*}\right) x, r, \theta ; x$ étant la distance des électrons à l'interface. Ces trajectoires sont décomposées en une succession de parcours élémentaires effectués en des temps $\Delta t_{i}=t_{i+1}-t_{i}$ suffisamment courts pour que la condition :

$$
\left|\mathbf{v}_{i}\right| \Delta t_{i} \ll \lambda_{\mathrm{R}}
$$

soit satisfaite. ( $v_{i}$ est la vitesse de l'électron au début du parcours élémentaire d'indice $i$.) En pratique on a choisi : $\Delta t_{i}=2,5 \times 10^{-2} \lambda_{\mathrm{R}} /\left|\mathbf{v}_{i}\right|$ sauf pour le premier parcours dont la longueur est fixée à une valeur faible devant $\lambda_{R}$.

En l'absence de collision, $v_{x, i+1}$, la composante perpendiculaire à l'interface de la vitesse à la fin de l'intervalle de temps $\Delta t_{i}$, peut être calculée à partir de $v_{x, i}$ :

$$
v_{x, i+1}=v_{x, i}+q E\left(x_{i}\right) \Delta t_{i} / m_{\mathrm{e}}^{*}
$$

$x_{i}$ étant la distance de l'électron à l'interface au début de l'intervalle de temps $\Delta t_{i}$, et $E\left(x_{i}\right)$ le champ électrique à cette distance. La composante parallèle à l'interface $V_{r i}$ est au contraire conservée. On en déduit les accroissements élémentaires :

$$
\begin{gathered}
\Delta x_{i}=\Delta t_{i}\left[v_{x, i}+\frac{1}{2}\left(v_{x, i+1}-v_{x, i}\right)\right] \\
\Delta r_{i}=v_{r i} \Delta t_{i} .
\end{gathered}
$$

Compte tenu des valeurs de $\lambda_{\mathrm{R}}$ utilisées dans le calcul, la condition (I.1) permet de calculer la longueur $\Delta s_{i}$ de chaque parcours élémentaire en supposant que celui-ci est rectiligne :

$$
\Delta s_{i}=\left(\Delta x_{i}+\Delta r_{i}\right)^{1 / 2}
$$

Pour chaque parcours de longueur $\Delta s_{i}$ on effectue un tirage aléatoire prenant en considération les événements autorisés d'après l'énergie cinétique acquise par l'électron, afin de déterminer si une collision (électron-phonon ou ionisation d'impact) se produit.

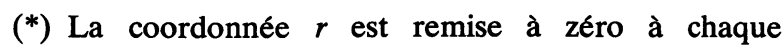
collision.
Puisque $\Delta s_{i} \ll \lambda_{\mathrm{R}}$ et $\lambda_{\mathrm{R}} \leqq \lambda_{\mathrm{i}}$, les probabilités correspondantes sont prises égales à $\Delta s_{i} / \lambda_{\mathrm{R}}$ et $\Delta s_{i} / \lambda_{\mathrm{i}}$, ou nulles si l'énergie est insuffisante. Le même tirage sert à déterminer la distance $x_{i+1}^{\prime}$, en général comprise entre $x_{i}$ et $x_{i+1}$, séparant le point de collision de l'interface. Si une collision électronphonon intervient, un second tirage aléatoire permet de déterminer, selon une loi de distribution de probabilité isotrope, l'orientation de la vitesse $V_{i+1}^{\prime}$ au début du parcours suivant. $\left|\mathbf{v}_{i+1}^{\prime}\right|$ est donné par :

$$
\frac{1}{2} m_{\mathrm{e}}^{*} \mathbf{v}_{i+1}^{\prime 2}=q\left[V(0)-V\left(x_{i+1}^{\prime}\right)\right]-n E_{\mathrm{R}} .
$$

Cette relation s'applique à la $n$-ième collision électron-phonon. $V(x)$ est le potentiel électrique à la distance $x$ de l'interface. On calcule alors le parcours suivant, selon le processus précédemment exposé. $\mathrm{Si}$ une collision avec ionisation d'impact intervient, on passe au calcul de la trajectoire de l'électron suivant, après avoir comptabilisé l'événement.

Dans les conditions retenues pour modéliser le comportement des varistances, la plupart des électrons perdent leur énergie par collision électronphonon avant de pouvoir créer une paire électrontrou. Le taux de génération, défini par le rapport du nombre de paires créées au nombre total d'électrons injectés à travers l'interface, est alors petit devant l'unité. Ceci impose de répéter le calcul sur un grand nombre d'électrons (jusqu'à 10 000) afin de réduire la dispersion des résultats.

Annexe II. - Calcul analytique d'un majorant du taux de création des paires électron-trous.

En partant des probabilités élémentaires $d x / \lambda_{R}$ et $\mathrm{d} x / \lambda_{\mathrm{i}}$ d'observer une collision électron-phonon et une création de paire électron-trou sur un intervalle de longueur $\mathrm{d} x$, on calcule les probabilités suivantes :

- la probabilité $p_{N}$ pour obtenir $N$ collisions électron-phonon dans une région de largeur $W_{\mathrm{i} N}$ où l'énergie cinétique de l'électron est inférieure à $E_{\mathrm{i}}$ :

$$
p_{N}=\frac{1}{N !}\left(\frac{W_{\mathrm{i} N}}{\lambda_{\mathrm{R}}}\right)^{N} \exp \left(-\frac{W_{\mathrm{i} N}}{\lambda_{\mathrm{R}}}\right)
$$

- la probabilité $q_{M}$ pour qu'un électron d'énergie cinétique $E>E_{\mathrm{i}}+M E_{\mathrm{R}}$ provoque une ionisation d'impact avant de subir $M$ collisions électron-phonon :

$$
q_{M}=1-\left(\frac{\lambda_{\mathrm{i}}}{\lambda_{\mathrm{i}}+\lambda_{\mathrm{R}}}\right)^{M}
$$

En sommant les probabilités $p_{N}$ il faut éviter de comptabiliser plusieurs fois la même combinaison d'événements. En particulier, il faut déduire de $p_{N}$ les probabilités $r_{n, N}$ d'obtenir $n$ collisions élec- 
tron-phonons dans l'intervalle $W_{\text {in }}$ et $N-n$ entre $W_{\text {in }}$ et $W_{\text {iN }}$ puisque celles-ci seront prises en compte dans le calcul de $p_{n}$ si $n<N$. D'après (II.1) :

$$
\begin{array}{r}
r_{n, N}=\frac{1}{n !(N-n) !} \frac{W_{\mathrm{i} n}^{n}\left(W_{\mathrm{i} N}-W_{\mathrm{i} n}\right)^{N-n}}{\lambda_{R}^{N}} \times \\
\times \exp \left(-\frac{W_{\mathrm{i} N}}{\lambda_{\mathrm{R}}}\right)
\end{array}
$$

- la probabilité $p_{N}$ d'obtenir $N$ collisions électron-phonons sur $W_{\mathrm{i} N}$ sans que cette succession d'événements soit comptabilisée pour $n<N$, et d'obtenir ensuite une ionisation d'impact, est alors :

$$
P_{N}=\left(p_{N}-\sum_{n=0}^{N-1} r_{n, N}\right) q_{M}
$$

avec $M=N_{\max }-N+1, N_{\max }$ étant la partie entière de $\left(q V_{2}-E_{\mathrm{i}}\right) / E_{\mathrm{R}}$. Le taux $G$ de création des paires électron-trou est alors donné par la sommation des probabilités $P_{N}$ pour les valeurs permises de $N$ :

$$
G=\sum_{N=0}^{N_{\max }} P_{N}
$$

ce qui correspond à la relation (18) du paragraphe 2.5 .

\section{Bibliographie}

[1] Matsuoka, M., Jpn. J. Appl. Phys. 10 (1970) 736.

[2] Levinson, L. M., Philipp, H. R., J. Appl. Phys. 46 (1975) 1332.

[3] Levine, J. D., CRC Crit. Rev. Sol. St. Sci. 5 (1975) 597.

[4] Guastavino, J., Moulanier, G., Congrès Electrotechnique Mondial (Moscou, 21-25 juin 1977).

[5] Emtage, P. R., J. Appl. Phys. 48 (1977) 4372.

[6] Bernasconi, J., Strassler, S., KNEChT, B., Klein, H. P., Menth, A., Solid State Commun. 21 (1977) 867.

[7] Mahan, G. D., Levinson, L. M., Philipp, H. R., J. Appl. Phys. 50 (1979) 2799.

[8] Hower, P. L., Gupta, T. K., J. Appl. Phys. 50 (1979) 4847.

[9] Pike, G. E., Grain Boundaries in Semiconductors (North-Holland, Amsterdam) (1982) 369.

[10] Buchy, F., Haussonne, J. M., American Ceramic Society, 81st annual metting, Cincinnati, Ohio (1979).

[11] van Kemenade, J. T. C., Eijnthoven, R. K., J. Appl. Phys. 50 (1979) 938.

[12] Mahan, G. D., Levinson, L. M., PhilipP, H. R., Appl. Phys. Lett. 33 (1978) 830.

[13] MANUEL, P., Journée d'études SEE : Varistances à base d'oxyde de zinc, 6 mars 1986 (Gif-surYvette), p. 25.

[14] Rossinelli, M., Blatter, G., Greuter, F., SCHMUECKLE, F., Journée d'études SEE : Varis- tances à base d'oxyde de zinc, 6 mars 1986 (Gifsur-Yvette) p. 58.

[15] Pike, G. E., Seager, C. H., J. Appl. Phys. 50 (1979) 3414 .

[16] Baraff, G. A., Phys. Rev. 128 (1962) 2507.

[17] Voir par exemple LogAN, R. A., White, H. G., J. Appl. Phys. 36 (1965) 3945.

[18] Schockley, W., Solid State Electron. 2 (1961) 35.

[19] Eun Dong KIM, Chong Hee KIM, MyUng Hwan OH, J. Appl. Phys. 58 (1985) 3231.

[20] Binesti, D., Thèse de Doctorat, Université de Bordeaux (1985) ;

Binesti, D., Bonnet, J. P., ONILlon, M., SAlmon, R., Rev. Int. Hautes Temp. Réfract. 23 (1986) 1.

[21] Kusy, A., KleinPenning, T. G. M., J. Appl. Phys. 54 (1983) 2900.

[22] Prudenziati, M., Masoero, A., Rietto, A. M., J. Appl. Phys. 58 (1985) 345.

[23] Vicaud, A., Rousseau, A., Hennebique, I., Conférence Internationale sur les Grands Réseaux Electriques (Paris, 1986) Rapport 33-10.

[24] Eda, K., J. Appl. Phys. 50 (1979) 4436.

[25] Philipp, H. R., Levinson, L. M., J. Appl. Phys. 50 (1979) 383.

[26] EINZINGER, R., Journée d'études SEE : Varistances à base d'oxyde de zinc, 6 mars 1986 (Gif-surYvette) p. 97.

[27] Philipp, H. R., Levinson, L. M., J. Appl. Phys. 48 (1977) 1621. 